
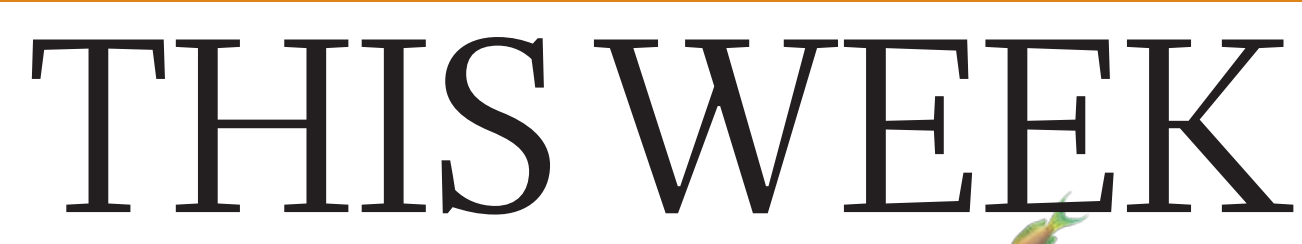

EDITORIALS

WORLD VIEW Why the US should not double its budget for basic science $\mathbf{p . 1 3 5}$
BENEFIT CUTS Blenny fish work for public good $\mathbf{p . 1 3 6}$

\section{.}

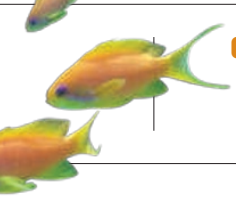

COMET Hartley 2 reveals its secrets in close encounter $\mathbf{p} \mathbf{. 1 3 8}$

\title{
Combating schizophrenia
}

\section{Research has revealed daunting complexities in the psychiatric condition, but also new routes towards diagnosis and treatment.}

S ome people hear voices that are the products only of processes in their brains. These hallucinations can lead to persistent delusions that someone is plotting against them or urging them to harm others. When a person acts on those delusions,

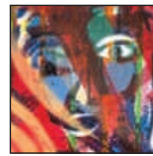

SCHIZOPHRENA

Search for origins and treatments nature.com/schizophrenia lack of coherent research and due priority (see page 165). In the current round of development of clinical diagnostics of the disease, the very definition of schizophrenia is under debate (see page 168).

One of the key diagnostic and scientific chalheadline-grabbing tragedy can ensue, usually involving someone close to the protagonist.

Violence is not a symptom of schizophrenia - only a tiny proportion of sufferers with the condition are homicidal. Yet these incidents dominate the media coverage of the disease. The reality of schizophrenia is much more complex. Hallucinations are one of several symptoms, others of which - cognitive dysfunction, loss of motivation and of social engagement - are much less amenable to medication, and are often more damaging to the ability of those with schizophrenia to function. In recent years it has been increasingly appreciated that this collection of symptoms, which typically first fully manifest in early adulthood, represents a late stage of the illness, and that the illness itself may perhaps turn out to be a collection of syndromes, rather than a single condition.

Motivated by the undue stigma and by the recent advances reported in our own pages and elsewhere, Nature this week examines the state of our understanding of schizophrenia, and how researchers can hope to make progress in an entangled landscape of innate and environmental influences. The image on this issue's cover and in the logo that links the associated content is a piece of art by a schizophrenia sufferers. It is one of many compiled by NARSAD, a US charity based in New York that spends significant public donations on psychiatric health research. The image reflects a world of confusion and distorted reality — but not a 'split personality', which is a mythical symptom of the condition, and leads to a misleading metaphorical use of the word 'schizophrenic' that those involved with the condition perpetually seek to eradicate.

Globally, somewhere between $0.5 \%$ and $1 \%$ of the population will experience schizophrenia in their lifetime. It is not the most prevalent psychiatric condition - clinical depression, aka unipolar disorder, is the most common. But analyses by the World Health Organization and others show that it has a disproportionate social cost. Moreover, owing to the few high-profile tragic episodes, it is probably the psychiatric condition with the most stigma attached - a stigma that is persistent, as those who have fought it have discovered time and again (see page 163).

In some societies, schizophrenia is not recognized as a disease - the response to its symptoms can be very dependent on culture, as can the degree of support that sufferers receive. Where it is recognized and treatment is given, it poses some of the greatest challenges in medical care. Drugs that treat the delusional symptoms have been around for decades, and have helped many sufferers enormously. But these drugs have not advanced in effectiveness, and several manufacturers have now backed out of this arena (see page 158). Cognitive and behavioural therapies have also made progress, but they suffer from a lenges is to distinguish the early phases of this condition, using all the means of biological, behavioural and cognitive analyses at our disposal. As with most diseases, the earlier the diagnosis, the greater the ability to mitigate the disease's impact or to defeat it. Recent research indicates that it may be possible to diagnose an 'at-risk' condition at the earliest phases of schizophrenia - for that reason as well as others, studies of adolescent brain development and behaviour have become a crucial research pathway (see pages 154 and 187). More generally, studies of the brain's morphology and circuitry (see page 194), and of the ways in which genetic and environmental influence interact (see page 203), are poised to make substantive progress.

We are in a decade in which fundamental science and clinical and social research can make an enormous impact on mental health (see Nature 463, 9; 2010). Those who have schizophrenia, and their families and colleagues who are also affected by the condition, depend on research to deliver new diagnostics and treatments. The rapid progress in science is daunting in the complexities that it has revealed. But it is yielding much for sufferers to hope for and new paths for researchers to follow. Funders please note.

\section{States or the union}

\section{California climate initiative moves decisively forward, providing a glimmer of hope.}

$\mathrm{B}$ efore the Democrats took control of Congress in 2006 and the White House in 2008, US climate strategists focused much of their energy at state and local level. Their goal was threefold: to test new climate policies, to reduce greenhouse-gas emissions where possible and to increase pressure on businesses and policy-makers to craft a viable national compromise. The third strand of that strategy failed this year, making the successes attained in the other two all the more important. From this perspective, the vital environmental vote of last week's midterm elections was in California, which forcefully rejected the ballot aiming to scuttle the state's pioneering climate law, Proposition 23.

Sixty-one per cent of California voters weighed in against Proposition 23, which put forward short-sighted arguments that the state's drive to reduce greenhouse-gas emissions would hobble an already 